\title{
$\bullet$ To Study the Effects of Viral Diseases on the
Human Body and Their Effective Treatment
}

\section{IJCRR}

Section: Healthcare

Sci. Journal Impact

Factor: 6.1 (2018)

ICV: 90.90 (2018)

\section{Rahmanova Sanobar Sabirovna}

Candidate of natural Science, Docent, TMA Urgench branch head of the Department, Propaedeutics of Internal Diseases, Uzbekistan.

\section{ABSTRACT}

Viruses are microorganisms that multiply only in living cells and cause infections in plants, animals, and humans. In the past, the term "virus" has been used to refer to various pathogens, especially unknown agents. After the French scientist, L. Pasteur proved the role of bacteria in the origin of several diseases, the concept of the virus began to be used as a synonym for the word "microbe". When we test viruses in the laboratory, they break down at the expense of amino acids in the cytoplasm, and it needs several amino acids. When we look at a set of virus molecules under a microscope, they look like crystals or X-cells. It is believed that their reproduction can take place around them. When we studied viruses, we found that they spread in several different ways. Studies have shown that most viruses are spread by airborne droplets. One of the main principles of combating infectious diseases is early detection and prevention of the spread of the disease. This is a very complex job and largely depends on the experience and qualifications of the specialist. In addition to the traditional methods used in other areas, some special methods are used in the detection of infectious diseases.

Key Words: Bacteria, Living cells, Viruses, Ecology, Infectious diseases, Vaccines, Influenza, Diagnostics

\section{INTRODUCTION}

The virus is widespread and causes a variety of serious diseases in humans, animals and plants. They are spread by a special distributor or mechanically. Most viruses do not lose their viability over the years but become infected as soon as they are exposed to the right conditions (living cells). Some viruses lose their properties in the external environment. Often, only one virus particle can cause a viral infection. For example, from a single poliovirus molecule, billions of viruses are formed in a matter of hours. Reproduction of the virus is associated with amino acids in the cytoplasm. ${ }^{1}$ The sum of millions of virus molecules is visible under a microscope in the form of crystals or Xcells. The pathogenesis of the disease is that it is relatively small compared to other pathogens and does not develop in a normal artificial nutrient medium. Except for a few bacteriophages, they are lab. has been shown to reproduce under All viruses that can be studied in the laboratory are "measured" more accurately by various physical methods. Their diameter is 10-300 microns. It can be in the form of a stick, a ball or a string. Many virus pathogens that cause disease in plants and animals are round in shape. Wheat and alfalfa mosaic The virus looks like a bacterial rod or an arrow. The structure of the virus has been identified by electron microscopy and X-rays. They all contain an inner substance, mainly nucleic acid, which is surrounded by a protein shell. The chemical composition of only a few species of the virus has been studied. The structure of vaccines virus is probably as complex as that of ordinary bacteria. It contains nucleoproteins, carbohydrates and lipids. Nucleic acid occurs in the form of deoxyribose, and the lipid group occurs in the form of cholesterol, phospholipids, and neutral fats. Phytopathogenic VIRUS contains ribonucleic acid (RNA), while pathogenic VIRUS contains RNA or DNA (deoxyribonucleic acid). Some VIRUS is obtained in the form of purified preparations, some of which form pure true crystals (eg, VIRUS of tobacco necrosis), while others form liquid crystals (e.g., tobacco mosaic VIRUS) or shale sediments. Ultracentrifuged to separate and purify VIRUS, various physicochemical methods are used. ${ }^{2,3}$

The classification of viruses and the symbols that represent them have not yet been adopted. They are given the same species and genus names as animals and plants, folk expressions, various abbreviations, are called by the genus name

\section{Corresponding Author:}

Rahmanova Sanobar Sabirovna, Candidate of natural Science, Docent, TMA Urgench branch head of the Department, Propaedeutics of Internal Diseases, Uzbekistan; E-mail: ssr.rss2011@mail.ru

ISSN: $2231-2196$ (Print)

ISSN: 0975-5241 (Online)

Received: 17.07 .2020

Revised: 25.09 .2020

Accepted: 16.11 .2020

Published: 05.01 .2021 
of the diseased organism, numbered next to it, or grouped into seeds and families according to VIRUS morphological, chemical, and reproductive properties. The Latin name for the VIRUS genus includes the word virus (eg, enterovirus), and the family name includes the word viridae (eg, Poxviridae).

The virus enters the body in various ways, for example, the virus can enter plant cells from the outside only when they are damaged. Influenza virus and others contain enzymes that break down the cell membrane. When a virus enters the body, a latent or latent period of infection begins. Many viruses accumulate in cells and form specific components within the cell (see Viral granullosis). Plants infected with Virus usually become a source of infection throughout their lives. virus ecological, biological and bacteria. has a strong variability under the influence of factors. the virus is common in nature and has many hosts. It is mainly spread by sucking insects, canals and nematodes. Some virus is transmitted by seeds, and almost all virus-infected plants are passed on to offspring when they are asexually propagated. The pathological effects of the virus are varied, mainly due to the disruption of protein and nuclein metabolism in the host organism due to their proliferation (see Viral diseases) which is studied by virology.

\section{INFECTIOUS DISEASES}

Infectious diseases are diseases caused by pathogenic microorganisms (bacteria, viruses, the simplest animals, etc.) that multiply in humans, animals and plants and have harmful effects. Some infectious diseases (eg, measles) are transmitted by walking close to a patient, to whom the term "infectious disease" refers. Some infectious diseases (eg, malaria) are not transmitted by close contact ("contact") with the patient, which means that the term "infectious disease" is less appropriate for them.

The main symptoms of infectious diseases are the presence in the patient's body of a specific microbe that causes the disease and the fact that the disease can be transmitted from person to person. The real causes of infectious diseases were discovered in the second half of the 19th century. Some diseases (plague, diarrhoea, paratyphoid, dysentery, and other intestinal infections) are transmitted through the digestive tract (through water and food that have been excreted by patients, or through unwashed hands that have been touched by these faeces). Influenza, whooping cough, mumps, diphtheria, measles, and other diseases are caused by airborne particles (droplet infections) that are released when a patient coughs, sneezes, or talks. enters. Some diseases are transmitted by blood-sucking insects (lice, mosquitoes, fleas, mites, scabies, etc.) (malaria, rash sweating, recurrent sweating, tick-borne encephalitis, scabies fever, etc.). When walking close to the patient or his towel, dishes, etc. Infectious diseases (venereal diseases, anthrax, scabies, etc.) are a separate group. Infectious diseases can last for several days (influenza, measles, scabies) or weeks (diarrhoea, rash, etc.) or last for months or even years (tuberculosis, leprosy, ulcers). The origin of infectious diseases depends on the number of pathogenic microbes entering the body, virulence, location, age, susceptibility to infection, as well as the external environment of the microbe (in unfavourable conditions, the virulence of the microbe decreases). Social conditions (housing, diet, culture, health care) play a crucial role in the emergence and spread of infectious diseases. Depending on the interaction of these conditions, different forms of infectious diseases (typical - true, mild, etc.) appear. Infectious diseases differ in the incubation period, the period of onset and exacerbation of symptoms, the period of disease exacerbation, the period of extinction and the period of recovery. Each of the infectious diseases has the characteristics of these periods. Some infectious diseases, such as diarrhoea, cause the microbe to remain in the affected organism and be released into the environment. Immunity remains after many infectious diseases. For example, the diagnosis of infectious diseases is based on clinical signs of the disease, the results of laboratory tests and epidemiological data. Patients are treated in specially equipped infectious disease hospitals. Prevention plays a crucial role in the fight against infectious diseases.

\section{CHARACTERISTICS OF INFECTIOUS DISEASES}

To prevent the spread of infectious diseases, patients with or suspected of having such diseases are isolated in a hospital or at home. Tone (plague), plague (cholera), rash sweating (rash typhoid), diarrhoea (typhoid fever), paratyphoid, dysentery, viral hepatitis, diphtheria, etc. persons diagnosed or suspected of having the disease must be transported to the hospital in a special ambulance. Influenza, measles, pertussis, etc. In some cases, patients with infectious diseases can be isolated at home, provided they are kept in a separate room, provided with proper care and disinfection.

In sanatoriums, rest homes, children's health facilities, kindergartens and nurseries, as well as in hospitals for therapy, surgery, paediatrics, etc. (except for the infectious department) is equipped with an insulator. In particular, people who are close to patients with dangerous infections (plague, plague) should be isolated for a period equal to the incubation period of those diseases. In other infectious diseases, patients are isolated at different times

Infectious diseases have their characteristics. They include:

1. All infectious diseases can be transmitted from a patient or a carrier of bacteria to healthy people around. The probability of transmission of the disease to others depends on the type and course of the disease. 
2. Infectious diseases Each disease is characterized by a specific type of pathogenic microbe. For example, diphtheria is caused by the diphtheria bacillus, measles is caused by the measles virus, and plague is caused by the plague (when the plague does not cause diphtheria or vice versa).

3. There is a certain periodicity in the course of infectious diseases. Once the pathogen enters the human body, there are no symptoms for some time. It is called the latent (incubation) period of infectious disease. The duration of this period varies in different diseases. For example, a few hours to 2 days in the flu, 2-3 weeks in diarrhoea, and so on. The next period is the period when the symptoms of the disease appear. It reveals both the general symptoms of the disease and the specific clinical symptoms of each disease. These symptoms first appear (prodromal period), develop and peak, and after a certain period go away and disappear. As the symptoms of the disease begin to subside, the patient begins to feel more normal. This marks the beginning of a period of convalescence. It often ends with a period of healing. In some cases, the disease may worsen during this period.

In infectious diseases, "complete recovery" means not only the complete cessation of symptoms but also bacteriological healing. Because when the patient is completely cured of the disease, the release of pathogenic microbes from his body must also stop. The fact that pathogenic microbes are not detected 2-3 times in the analysis (ointment or implants) performed after the formation of the patient indicates bacteriological recovery. In some diseases, such as diarrhoea or paratyphoid, pathogenic microbes are released from the body even after the patient has recovered. It is called the post-disease bacterial carrier condition. If this condition lasts for up to 3 months, it is an acute bacterial carrier, and if it lasts more than 3 months, it is a chronic bacterial carrier.

4. After infectious diseases, the patient's body develops stagnant immunity to the germs of this disease. It is an acquired immunity and the ability to defend is maintained for various periods. For example, post-influenza immunity lasts up to 3 years against this type of virus. The immunity that develops after measles and diarrhoea lasts a lifetime and a person does not get sick with these diseases again. In recent years, scientific studies have shown that immunity formed after infectious diseases largely depends on the genetic or phenotypic characteristics of the diseased organism.

5. Common infectious diseases can be prevented through vaccination. The goal of vaccinating children against several infectious diseases is to prevent them. These include vaccines against diphtheria, pertussis, measles, and polio.

In some cases, the human body is indifferent to disease germs. The immune response to the microbe does not develop. There will be no symptoms of the disease. The microbe, on the other hand, is located in a known and average organ and can only be detected by laboratory tests. For example, diphtheria bacilli or meningococci may be present in a person's throat for some time, but may not have symptoms of the disease. This condition is called bacterial overdose. In humans, the pathogen is found only once, and if the disease is not observed, it is considered to be a transient bacterium.

In some stages of infectious disease, germs that enter the body can also enter the bloodstream. This condition is called bacteremia. In some diseases, the patient takes venous blood from the vein to look for germs, that is, to use it in diagnostics. For example, bacteremia occurs in typhoid fever. Therefore, to make a diagnosis, among other tests, blood is taken from the wrist and injected into the bile fluid. Later, the disease microbe can be found in it.

\section{PERSPECTIVE}

Although infectious diseases are caused by pathogenic bacteria, viruses, and simple single-celled organisms, their occurrence cannot be considered solely as a result of the microbe's fight against the organism. The outbreak is a complex social biological process that depends on the interaction of the microbe with the macroorganism. When a pathogenic bacterium enters, the human body undergoes pathological changes, adaptation and defence processes, i.e. the development of an infectious disease. Once a pathogenic microbe enters the body, the disease does not have to develop. The relationship between a pathogenic microbe and the human body can be different, depending on the virulence of the microbe on the one hand and the susceptibility and reactivity of the human body to the disease on the other hand..$^{3-5}$

Infectious diseases differ from other diseases by the following 4 features:

1. Infectious disease is caused by a live pathogenic microbe.

2. The patient in turn becomes the source of the disease and can spread it to others.

3. Whichever infectious disease a patient suffers from, his body develops immunity against that disease and resists the re-transmission of that disease.

4. Infectious diseases develop and disappear with certain periods, i.e. cyclically.

The pathogenic microbe tries to protect itself from any opposing forces, under small conditions it begins to multiply rapidly, adapts to phagocytes (forms a capsule, produces substances such as aggression, atifagin, virulinka). the ability of a microorganism to overcome its protective mechanisms and show its harmful effects is its virulence. ${ }^{6,7}$ 
Epidemiology is the study of the laws of the emergence, spread, and spread of infectious diseases and the development of measures to combat them. The term epidemiology is derived from the Latin epi-many, Demas-population, meaning the spread of disease within a population. Depending on the number of people infected with the infectious disease, there are different types of epidemiological processes.

1. Sporadic diseases. People with the disease are rare.

2. An epidemic is the spread of an infectious disease in any country, province or country.

3 A pandemic is the simultaneous spread of an infectious disease (eg, cholera, influenza) on an international scale, ie in several countries and continents.

4. Endemicity - the constant occurrence of any infectious disease in a particular area.

5. Enzootia - the constant occurrence of an infectious disease (eg, plague, leishmaniasis) among animals living somewhere (for example, rodents)

6. Epizootics - the spread of any infectious disease among animals.

7. Exotic diseases - infectious diseases imported from abroad. ${ }^{4,6,7}$

Infectious diseases are divided into two groups depending on the source of infection: 1. Anthropozoonoses - these diseases occur only in humans and do not infect animals (typhoid, diarrhoea, hepatitis, AIDS). Zoonoses - these diseases are in animals and humans occurs. Infection in humans is transmitted from animals (brucellosis, plague, rabies, anthrax).

\section{CONCLUSION}

It is less difficult to diagnose during an infectious disease outbreak. During this period, the specific clinical symptoms of each disease are clearly expressed. In addition to the patient's life history, the history of the disease is also inquired. Whenever possible, determine when and with what symptoms the disease has started. In the following days, they are asked what symptoms were added to them and how the initial symptoms developed. The patient himself may not know what is worth noting in the anamnesis for the medical professional. Therefore, it is necessary to get the necessary information from him through questions. In particular, it is important to know when the body temperature has risen, how many degrees it has reached, and at what time of the day it is most pronounced. Headaches, sleep disturbances are not only common symptoms but also specific to some infectious diseases. It is even important to know where the head hurts. Information such as the appearance of rashes on the body, their appearance, in what order they rash, how long they persist, also helps to determine the true nature of the disease. In the diagnosis of intestinal infections, information such as abdominal pain, where it is most often felt, nausea, vomiting, diarrhoea, recurrence of these symptoms make it easier to diagnose.

\section{Conflict of interest: None}

\section{Financial support: None}

\section{REFERENCES}

1. Kubanova AA, Martynov AA, Pirogova EV. Questions of Informatization in dermatovenerology. Collection of abstracts of the 10th anniversary scientific and practical conference of young scientists. Clin Expt Med 2010; 3:18-19.

2. Martynov AA, Pirogova EV. Telecommunication technologies in the process of providing specialized dermatovenerological care. Theses of the XIII International Congress of the interregional public organization.Society for pharmacoeconomic research. Justice Quality Economy Clin Pharmac Pharma 2010; 5:34

3. Morozova EV, Cartagena OB. medico-organizational measures to improve medical care on the profile of dermatovenereology in the Samara region. Mod Prob Sci Edu 2016;5:231.

4. Pirogova EV, Martynov AA. the current state of information infrastructure of specialized dermatovenerological institutions of the Russian Federation. Top Iss Health Org Dev 2011;136-145.

5. Ju Y, Yang MS. Asthma Simulation Team Experience Using Hybrid Modeling. Int J Cur Res Rev 2020;12(19):10.

6. Kurz H, Riedler J. An increase in allergic diseases in childhood-current hypotheses and possible prevention. Wiener medizinische Wochenschrift (1946). 2003;153(3-4):50-8.

7. Lapik SV, Zhmurov VA. Clinico-biochemical effectiveness of emoxpine in patients with bacterial bronchial asthma. Terapevticheskii arkhiv. 1998;70(11):72-4 